\title{
A New Route to Organoboron Polymers via Highly Selective Polymer Modification Reactions
}

\author{
Yang Qin, Guanglou Cheng, Obianuju Achara, Kshitij Parab, and Frieder Jäkle* \\ Department of Chemistry, Rutgers University-Newark, 73 Warren Street, Newark, NJ 07102
}

\section{Supplementary Material}

The labeling schemes on the right are used for ${ }^{1} \mathrm{H}$ and ${ }^{13} \mathrm{C}$ NMR assignments.

The abbreviations $\mathrm{Ph}$, Th, and $\mathrm{Pf}$ are used for phenyl, 2-thienyl, and pentafluorophenyl, respectively.
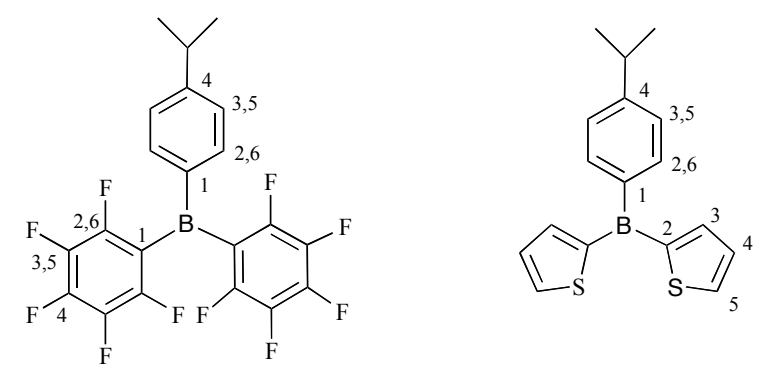

\section{Synthesis and Spectroscopic Data of Cumene Model Compounds}

Synthesis of 4-Trimethylsilylcumene ${ }^{1}$. 4-Bromocumene (10.44 g, $\left.52.4 \mathrm{mmol}\right)$ was dissolved in $200 \mathrm{ml}$ diethyl ether at $0{ }^{\circ} \mathrm{C}$ and $n$-BuLi $(36 \mathrm{ml}, 1.6 \mathrm{M}$ in hexanes, $58 \mathrm{mmol}$ ) was added dropwise. The mixture was allowed to warm to room temperature, stirred for $2 \mathrm{~h}$ and then cooled down to $-78{ }^{\circ} \mathrm{C}$. Neat $\mathrm{Me}_{3} \mathrm{SiCl}(8.6 \mathrm{ml}, 68 \mathrm{mmol})$ was added dropwise, and the mixture was warmed to room temperature, stirred overnight, and then poured into ice water containing $1 \%$ $\mathrm{NaOH}$. After standard workup and removal of all volatile components under vacuum at room temperature, the product was distilled at $43^{\circ} \mathrm{C}$ under high vacuum. 4-Trimethylsilylcumene was obtained as a colorless liquid (7.7 g, 76\%). ${ }^{1} \mathrm{H}$ NMR (399.952 MHz, $\left.\mathrm{C}_{6} \mathrm{D}_{6}\right) \square=7.47\left(\mathrm{~d},{ }^{3} \mathrm{~J}_{\mathrm{HH}}=8.0\right.$ $\mathrm{Hz}, 2 \mathrm{H}, \mathrm{Ph}-\mathrm{H} 2,6), 7.16\left(\mathrm{~d},{ }^{3} \mathbf{J}_{\mathrm{HH}}=8.0 \mathrm{~Hz}, 2 \mathrm{H}, \mathrm{Ph}-\mathrm{H} 3,5\right), 2.73$ (septet, ${ }^{3} \mathbf{J}_{\mathrm{HH}}=7.2 \mathrm{~Hz}, 1 \mathrm{H}$, $\left.\mathrm{C} \boldsymbol{H}\left[\mathrm{CH}_{3}\right]_{2}\right), 1.16\left(\mathrm{~d},{ }^{3} \mathrm{~J}_{\mathrm{HH}}=7.2 \mathrm{~Hz}, 6 \mathrm{H}, \mathrm{CH}\left[\mathrm{CH}_{3}\right]_{2}\right), 0.24\left(\mathrm{~s}, 9 \mathrm{H}, \mathrm{Si}\left[\mathrm{CH}_{3}\right]_{3}\right) ;{ }^{13} \mathrm{C}$ NMR $(100.564$ $\left.\mathrm{MHz}, \mathrm{C}_{6} \mathrm{D}_{6}\right): \square=149.6$ (Ph-C4), 137.3 (Ph-C1), 133.8 (Ph-C2,6), 126.3 (Ph-C3,5), 34.4 $\left(\mathrm{CH}\left[\mathrm{CH}_{3}\right]_{2}\right), 24.1\left(\mathrm{CH}\left[\mathrm{CH}_{3}\right]_{2}\right),-0.9\left(\mathrm{Si}\left[\mathrm{CH}_{3}\right]_{3}\right) ;{ }^{29} \mathrm{Si} \mathrm{NMR}\left(79.4 \mathrm{MHz}, \mathrm{C}_{6} \mathrm{D}_{6}\right)$ : $\square=-4.3$; GC-MS (t $=5.7 \mathrm{~min}): \mathrm{m} / \mathrm{z}: 192\left[\mathrm{M}^{+}\right], 177\left[\mathrm{M}^{+}-\mathrm{CH}_{3}\right]$.

Synthesis of 4-Dibromoborylcumene. A solution of $\mathrm{BBr}_{3}(4.85 \mathrm{~g}, 19.4 \mathrm{mmol})$ in $5 \mathrm{ml} \mathrm{CH}_{2} \mathrm{Cl}_{2}$ was added dropwise to a solution of 4-trimethylsilylcumene $(3.38 \mathrm{~g}, 17.6 \mathrm{mmol})$ in $10 \mathrm{ml}$ $\mathrm{CH}_{2} \mathrm{Cl}_{2}$. The reaction mixture was stirred for $2 \mathrm{~h}$, and all volatile material was subsequently 
removed under reduced pressure $\left(10^{-3}\right.$ torr). The crude product was distilled at $56{ }^{\circ} \mathrm{C}$ under high vacuum to give 4-dibromoborylcumene as a colorless liquid with a yield of $4.88 \mathrm{~g}(96 \%) .{ }^{1} \mathrm{H}$ NMR (399.952 MHz, $\left.\mathrm{C}_{6} \mathrm{D}_{6}\right): \square=8.08\left(\mathrm{~d},{ }^{3} \mathrm{~J}_{\mathrm{HH}}=8.0 \mathrm{~Hz}, 2 \mathrm{H}, \mathrm{Ph}-\mathrm{H} 2,6\right), 6.92\left(\mathrm{~d},{ }^{3} \mathrm{~J}_{\mathrm{HH}}=8.0 \mathrm{~Hz}, 2 \mathrm{H}\right.$, $\mathrm{Ph}-\mathrm{H} 3,5), 2.53$ (heptet, $\left.{ }^{3} \mathrm{~J}_{\mathrm{HH}}=7.2 \mathrm{~Hz}, 1 \mathrm{H}, \mathrm{CH}\left[\mathrm{CH}_{3}\right]_{2}\right), 0.99\left(\mathrm{~d},{ }^{3} \mathrm{~J}_{\mathrm{HH}}=7.2 \mathrm{~Hz}, 6 \mathrm{H}, \mathrm{CH}[\mathrm{CH}]_{2}\right) ;{ }^{1} \mathrm{H}$ NMR (399.952 MHz, $\left.\mathrm{CDCl}_{3}\right): \square=8.07\left(\mathrm{~d},{ }^{3} \mathrm{~J}_{\mathrm{HH}}=8.0 \mathrm{~Hz}, 2 \mathrm{H}, \mathrm{Ph}-\mathrm{H} 2,6\right), 7.23\left(\mathrm{~d},{ }^{3} \mathrm{~J}_{\mathrm{HH}}=8.0 \mathrm{~Hz}\right.$, $2 \mathrm{H}, \mathrm{Ph}-\mathrm{H} 3,5), 2.87$ (heptet, $\left.{ }^{3} \mathrm{~J}_{\mathrm{HH}}=7.2 \mathrm{~Hz}, 1 \mathrm{H}, \mathrm{CH}\left[\mathrm{CH}_{3}\right]_{2}\right), 1.19\left(\mathrm{~d},{ }^{3} \mathrm{~J}_{\mathrm{HH}}=7.2 \mathrm{~Hz}, 6 \mathrm{H}, \mathrm{CH}\left[\mathrm{CH}_{3}\right]_{2}\right)$; ${ }^{13} \mathrm{C}$ NMR (100.564 MHz, $\left.\mathrm{C}_{6} \mathrm{D}_{6}\right): \square=157.2$ (Ph-C4), 138.5 (Ph-C2,6), 126.6 (Ph-C3,5), 34.6 $\left(\boldsymbol{C H}\left[\mathrm{CH}_{3}\right]_{2}\right), 23.3\left(\mathrm{CH}\left[\mathrm{CH}_{3}\right]_{2}\right), \mathrm{Ph}-\mathrm{C} 1$ not observed; ${ }^{11} \mathrm{~B}$ NMR $\left(160.370 \mathrm{MHz}, \mathrm{C}_{6} \mathrm{D}_{6}\right): \square=56\left(\mathrm{w}_{1 / 2}\right.$ $=300 \mathrm{~Hz}$ ); EI-MS (\%): m/z: $290\left[\mathrm{M}^{+}\right](40), 275\left[\mathrm{M}^{+}-\mathrm{CH}_{3}\right]$ (100); elemental analysis: calcd. C $37.30 \mathrm{H} \mathrm{3.83}$; found C $38.06 \mathrm{H} 3.85$.

Synthesis of 4-Diethoxyborylcumene. $\mathrm{Neat} \mathrm{Me}_{3} \mathrm{SiOEt}(0.65 \mathrm{ml}, 4.2 \mathrm{mmol})$ was added dropwise to a solution of 4-dibromoborylcumene $(0.50 \mathrm{~g}, 1.73 \mathrm{mmol})$ in $\mathrm{CH}_{2} \mathrm{Cl}_{2}(10 \mathrm{ml})$ at room temperature. The mixture was stirred for $1 \mathrm{~d}$, and all volatile material was subsequently removed under reduced pressure ( $10^{-3}$ torr). 4-Diethoxyborylcumene was obtained as a colorless liquid in a yield of $0.37 \mathrm{~g}(98 \%)$ with $>95 \%$ spectroscopic purity as confirmed by ${ }^{1} \mathrm{H}$ NMR analysis. ${ }^{1} \mathrm{H}$ NMR (399.952 MHz, $\left.\mathrm{C}_{6} \mathrm{D}_{6}\right): \square=7.75\left(\mathrm{~d},{ }^{3} \mathrm{~J}_{\mathrm{HH}}=8.0 \mathrm{~Hz}, 2 \mathrm{H}, \mathrm{Ph}-\mathrm{H} 2,6\right), 7.17\left(\mathrm{~d},{ }^{3} \mathrm{~J}_{\mathrm{HH}}=8.0 \mathrm{~Hz}, 2 \mathrm{H}\right.$, $\mathrm{Ph}-\mathrm{H} 3,5$ ), 4.02 (quartet, ${ }^{3} \mathrm{~J}_{\mathrm{HH}}=7.2 \mathrm{~Hz}, 4 \mathrm{H}, \mathrm{OCH}_{2} \mathrm{CH}_{3}$ ), 2.73 (heptet, ${ }^{3} \mathbf{J}_{\mathrm{HH}}=7.2 \mathrm{~Hz}, 1 \mathrm{H}$, $\left.\mathrm{CH}\left[\mathrm{CH}_{3}\right]_{2}\right), 1.16\left(\mathrm{~d},{ }^{3} \mathrm{~J}_{\mathrm{HH}}=7.2 \mathrm{~Hz}, 6 \mathrm{H}, \mathrm{CH}\left[\mathrm{CH}_{3}\right]_{2}\right), 1.14\left(\mathrm{t},{ }^{3} \mathrm{~J}_{\mathrm{HH}}=7.2 \mathrm{~Hz}, 6 \mathrm{H}, \mathrm{OCH}_{2} \mathrm{CH}_{3}\right) ;{ }^{13} \mathrm{C}$ NMR (100.564 MHz, $\mathrm{C}_{6} \mathrm{D}_{6}$ ): $\square=150.4$ (Ph-C4), 134.3 (Ph-C2,6), 131.0 (br, Ph-C1), 126.0 $(\mathrm{Ph}-\mathrm{C} 3,5), 60.2\left(\mathrm{OCH}_{2} \mathrm{CH}_{3}\right), 34.5\left(\boldsymbol{C H}\left[\mathrm{CH}_{3}\right]_{2}\right), 24.0\left(\mathrm{CH}\left[\boldsymbol{C H}_{3}\right]_{2}\right), 17.7\left(\mathrm{OCH}_{2} \boldsymbol{C H}_{3}\right) ;{ }^{11} \mathrm{~B}$ NMR $\left(160.370 \mathrm{MHz}, \mathrm{C}_{6} \mathrm{D}_{6}\right): \square=28\left(\mathrm{w}_{1 / 2}=450 \mathrm{~Hz}\right)$; GC-MS $(\mathrm{t}=7.21 \mathrm{~min}): \mathrm{m} / \mathrm{z}: 220\left[\mathrm{M}^{+}\right], 205\left[\mathrm{M}^{+}-\right.$ $\mathrm{CH}_{3}$ ], 175, 135; (due to partial hydrolysis on the GC-MS column an additional band for the boroxin $(i \mathrm{PrPhBO})_{3}$ is observed at a higher retention time of $\left.14.8 \mathrm{~min}\right)$; elemental analysis: calcd. C 70.94 H 9.62; found C 69.98 H 9.50.

Synthesis of 4-Bis(4'-bromobutoxy)borylcumene. A solution of THF (1.6 ml, $20 \mathrm{mmol})$ in 5 $\mathrm{ml} \mathrm{CH} \mathrm{Cl}_{2}$ was added dropwise to a solution of 4-dibromoborylcumene $(0.25 \mathrm{~g}, 0.86 \mathrm{mmol})$ in $\mathrm{CH}_{2} \mathrm{Cl}_{2}(10 \mathrm{ml})$ at room temperature and the mixture was kept stirring for $2 \mathrm{~h}$. All volatile material was removed under reduced pressure $\left(10^{-3}\right.$ torr $)$. Distillation of the crude product at 90 ${ }^{\circ} \mathrm{C}$ under high vacuum gave $0.32 \mathrm{~g}(86 \%)$ 4-bis(4'-bromobutoxy)borylcumene as a colorless liquid. ${ }^{1} \mathrm{H}$ NMR $\left(399.952 \mathrm{MHz}, \mathrm{C}_{6} \mathrm{D}_{6}\right)$ : $\square=7.70\left(\mathrm{~d},{ }^{3} \mathrm{~J}_{\mathrm{HH}}=8.0 \mathrm{~Hz}, 2 \mathrm{H}, \mathrm{Ph}-\mathrm{H} 2,6\right), 7.20\left(\mathrm{~d},{ }^{3} \mathrm{~J}_{\mathrm{HH}}=\right.$ $8.0 \mathrm{~Hz}, 2 \mathrm{H}, \mathrm{Ph}-\mathrm{H} 3,5), 3.84\left(\mathrm{t},{ }^{3} \mathrm{~J}_{\mathrm{HH}}=6.4 \mathrm{~Hz}, 4 \mathrm{H}, \mathrm{OCH}_{2} \mathrm{CH}_{2} \mathrm{CH}_{2} \mathrm{CH}_{2} \mathrm{Br}\right), 2.96\left(\mathrm{t},{ }^{3} \mathrm{~J}_{\mathrm{HH}}=6.4 \mathrm{~Hz}\right.$, $4 \mathrm{H}, \mathrm{OCH}_{2} \mathrm{CH}_{2} \mathrm{CH}_{2} \mathrm{CH}_{2} \mathrm{Br}$ ), 2.73 (septet, $\left.{ }^{3} \mathrm{~J}_{\mathrm{HH}}=7.2 \mathrm{~Hz}, 1 \mathrm{H}, \mathrm{CH}\left[\mathrm{CH}_{3}\right]_{2}\right), 1.62,1.47(\mathrm{~m}, 8 \mathrm{H}$, $\left.\mathrm{OCH}_{2} \mathrm{CH}_{2} \mathrm{CH}_{2} \mathrm{CH}_{2} \mathrm{Br}\right), 1.16\left(\mathrm{~d},{ }^{3} \mathrm{~J}_{\mathrm{HH}}=7.2 \mathrm{~Hz}, 6 \mathrm{H}, \mathrm{CH}\left[\mathrm{CH}_{3}\right]_{2}\right) ;{ }^{13} \mathrm{C}$ NMR $\left(100.564 \mathrm{MHz}, \mathrm{C}_{6} \mathrm{D}_{6}\right)$ : $\square=150.7$ (Ph-C4), 134.3 (Ph-C2,6), 131.0 (br, Ph-C1), 126.1 (Ph-C3,5), 63.6 $\left(\mathrm{OCH}_{2} \mathrm{CH}_{2} \mathrm{CH}_{2} \mathrm{CH}_{2} \mathrm{Br}\right), \quad 34.5 \quad\left(\boldsymbol{C H}\left[\mathrm{CH}_{3}\right]_{2}\right), \quad 33.5 \quad\left(\mathrm{OCH}_{2} \mathrm{CH}_{2} \mathrm{CH}_{2} C_{2} \mathrm{Br}\right), \quad 30.4, \quad 29.6$ $\left(\mathrm{OCH}_{2} \boldsymbol{C H}_{2} \boldsymbol{C H}_{2} \mathrm{CH}_{2} \mathrm{Br}\right), 24.0\left(\mathrm{CH}\left[\mathrm{CH}_{3}\right]_{2}\right) ;{ }^{11} \mathrm{~B} \mathrm{NMR}\left(160.370 \mathrm{MHz}, \mathrm{C}_{6} \mathrm{D}_{6}\right): \square=28\left(\mathrm{w}_{1 / 2}=550\right.$ $\mathrm{Hz}$ ); GC-MS ( $\mathrm{t}=12.2 \mathrm{~min}): \mathrm{m} / \mathrm{z}: 434\left[\mathrm{M}^{+}\right], 419\left[\mathrm{M}^{+}-\mathrm{CH}_{3}\right], 233,205,135,55$ (due to partial hydrolysis on the GC-MS column an additional band for the boroxin $(i \mathrm{PrPhBO})_{3}$ is observed at a higher retention time of $14.8 \mathrm{~min}$ ); elemental analysis: calcd. C $47.05 \mathrm{H} 6.27$; found $\mathrm{C} 46.65 \mathrm{H}$ 6.46 .

Synthesis of 4-(Pinacolatoboryl)cumene. A solution of pinacol $(0.16 \mathrm{~g}, 1.36 \mathrm{mmol})$ in $10 \mathrm{ml}$ $\mathrm{CH}_{2} \mathrm{Cl}_{2}$ was added dropwise to a solution of 4-diethoxyborylcumene $(0.30 \mathrm{~g}, 1.36 \mathrm{mmol})$ in 10 $\mathrm{ml} \mathrm{CH}_{2} \mathrm{Cl}_{2}$ and the mixture was stirred at room temperature for $48 \mathrm{~h}$. All volatile material was removed under vacuum and the remaining solid was sublimed under high vacuum at $50-60{ }^{\circ} \mathrm{C}$ to give pinacolatoborylcumene as a white solid $\left(0.20 \mathrm{~g}, 59 \%\right.$ yield). ${ }^{1} \mathrm{H}$ NMR $(499.893 \mathrm{MHz}$, $\left.\mathrm{CDCl}_{3}\right) \square=7.76\left(\mathrm{~d},{ }^{3} \mathrm{~J}_{\mathrm{HH}}=8.5 \mathrm{~Hz}, 2 \mathrm{H}, \mathrm{Ph}-\mathrm{H} 2,6\right), 7.25\left(\mathrm{~d},{ }^{3} \mathrm{~J}_{\mathrm{HH}}=8.5 \mathrm{~Hz}, 2 \mathrm{H}, \mathrm{Ph}-\mathrm{H} 3,5\right), 2.92$ 
(septet, $\left.{ }^{3} \mathrm{~J}_{\mathrm{HH}}=6.5 \mathrm{~Hz}, 1 \mathrm{H}, \mathrm{CH}\left[\mathrm{CH}_{3}\right]_{2}\right), 1.34\left(\mathrm{~s}, 12 \mathrm{H}, \mathrm{O}\left[\mathrm{CH}_{3}\right]_{2} \mathrm{CC}\left[\mathrm{CH}_{3}\right]_{2} \mathrm{O}\right), 1.26\left(\mathrm{~d},{ }^{3} \mathrm{~J}_{\mathrm{HH}}=6.5 \mathrm{~Hz}\right.$, $\left.6 \mathrm{H}, \mathrm{CH}\left[\mathrm{CH}_{3}\right]_{2}\right) ;{ }^{13} \mathrm{C} \mathrm{NMR}\left(125.698 \mathrm{MHz}, \mathrm{CDCl}_{3}\right) \square=152.5(\mathrm{Ph}-\mathrm{C} 4), 135.2(\mathrm{Ph}-\mathrm{C} 2,6), 126.1$ $\left.(\mathrm{Ph}-\mathrm{C} 3,5), 83.8\left(\mathrm{O}_{[} \mathrm{CH}_{3}\right]_{2} \boldsymbol{C} \boldsymbol{C}\left[\mathrm{CH}_{3}\right]_{2} \mathrm{O}\right), 34.6\left(\boldsymbol{C H}\left[\mathrm{CH}_{3}\right]_{2}\right), 25.1\left(\mathrm{O}\left[\boldsymbol{C} \mathrm{H}_{3}\right]_{2} \mathrm{CC}\left[\boldsymbol{C} \mathrm{H}_{3}\right]_{2} \mathrm{O}\right), 24.1$ $\left(\mathrm{CH}\left[\mathrm{CH}_{3}\right]_{2}\right)$, Ph-C1 was not resolved; ${ }^{11} \mathrm{~B} \mathrm{NMR}\left(160.386 \mathrm{MHz}, \mathrm{CDCl}_{3}\right) \square=31\left(\mathrm{w}_{1 / 2}=320 \mathrm{~Hz}\right)$; GC-MS ( $\mathrm{t}=8.1 \mathrm{~min}): \mathrm{m} / \mathrm{z}: 246\left[\mathrm{M}^{+}\right], 231\left[\mathrm{M}^{+}-\mathrm{CH}_{3}\right], 203,160,147,131$, 105; elemental analysis: calcd. C 73.19 H 9.42; found C 73.28 H 9.75.

Synthesis of 4-Bis(2-thienyl)borylcumene. To a solution of 4-dibromoborylcumene (1.00 g, $3.45 \mathrm{mmol})$ in $\mathrm{CH}_{2} \mathrm{Cl}_{2}(10 \mathrm{ml})$ was added dropwise a solution of 2-trimethylstannylthiophene $(1.70 \mathrm{~g}, 6.90 \mathrm{mmol})$ in $\mathrm{CH}_{2} \mathrm{Cl}_{2}(10 \mathrm{ml})$. The mixture was stirred at room temperature for $2 \mathrm{~h}$, and all volatile material was subsequently removed under vacuum. High vacuum distillation $\left(145^{\circ} \mathrm{C}\right.$, $10^{-3}$ torr) gave compound M-BTh as colorless viscous oil $(0.88 \mathrm{~g}, 86 \%)$. ${ }^{1} \mathrm{H}$ NMR $(499.893$ $\mathrm{MHz}_{\mathrm{CDCl}}$ ): $\mathrm{\square}=8.05\left(\mathrm{~d},{ }^{3} \mathrm{~J}_{\mathrm{HH}}=4.8 \mathrm{~Hz}, 2 \mathrm{H}, \mathrm{Th}-\mathrm{H} 3\right), 7.97\left(\mathrm{~d},{ }^{3} \mathrm{~J}_{\mathrm{HH}}=3.5 \mathrm{~Hz}, 2 \mathrm{H}, \mathrm{Th}-\mathrm{H} 5\right), 7.90$ $\left(\mathrm{d},{ }^{3} \mathrm{~J}_{\mathrm{HH}}=8.0 \mathrm{~Hz}, 2 \mathrm{H}, \mathrm{Ph}-\mathrm{H} 2,6\right), 7.48\left(\mathrm{~m}, 4 \mathrm{H}\right.$, Th-H4 \& Ph-H3,5), 3.15 (septet, ${ }^{3} \mathrm{~J}_{\mathrm{HH}}=7.0 \mathrm{~Hz}, 1 \mathrm{H}$, $\left.\mathrm{CH}\left[\mathrm{CH}_{3}\right]_{2}\right), 1.49\left(\mathrm{~d},{ }^{3} \mathrm{~J}_{\mathrm{HH}}=7.0 \mathrm{~Hz}, 6 \mathrm{H}, \mathrm{CH}\left[\mathrm{CH}_{3}\right]_{2}\right) ;{ }^{13} \mathrm{C} \mathrm{NMR}\left(125.698 \mathrm{MHz}, \mathrm{CDCl}_{3}\right): \square=151.8$ (Ph-C4), 145.2 (br., Th-C2), 142.5 (Th-C3), 140.8 (br., Ph-C1), 137.4 (Ph-C2,6), 137.1 (Th-C5), 129.1 (Th-C4), $125.7(\mathrm{Ph}-\mathrm{C} 3,5), 34.4\left(\mathrm{CH}\left[\mathrm{CH}_{3}\right]_{2}\right), 24.1\left(\mathrm{CH}\left[\mathrm{CH}_{3}\right]_{2}\right) ;{ }^{11} \mathrm{~B} \mathrm{NMR}(128.286 \mathrm{MHz}$, $\left.\mathrm{CDCl}_{3}\right): \square=55\left(\mathrm{w}_{1 / 2}=1000 \mathrm{~Hz}\right) ; \mathrm{GC}-\mathrm{MS}(\mathrm{t}=11.5 \mathrm{~min}): \mathrm{m} / \mathrm{z}: 296\left[\mathrm{M}^{+}\right], 281\left[\mathrm{M}^{+}-\mathrm{CH}_{3}\right], 253$, 177.

Synthesis of 4-Bis(pentafluorophenyl)borylcumene. ${ }^{2,3}$ A solution of $\left[\mathrm{CuC}_{6} \mathrm{~F}_{5}\right]_{4}$ (toluene $)_{2}(1.00$ $\mathrm{g}, 0.90 \mathrm{mmol})$ in $\mathrm{CH}_{2} \mathrm{Cl}_{2}(10 \mathrm{ml})$ was added dropwise to a solution of 4-dibromoborylcumene

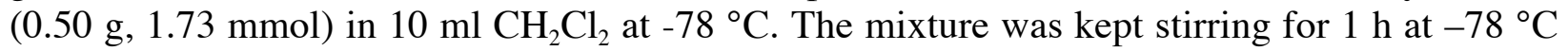
and then allowed to slowly warm to room temperature. A solid precipitate $(\mathrm{CuBr})$ formed and was removed by filtration through a fritted glass disk. All volatile material was removed under reduced pressure $\left(10^{-3}\right.$ torr $)$ to give a sticky white solid. The spectroscopic purity of the crude product was estimated to ca. $95 \%$ by ${ }^{1} \mathrm{H}$ and ${ }^{19} \mathrm{~F} \mathrm{NMR}$ analysis. Distillation of the crude product at $100{ }^{\circ} \mathrm{C}$ under high vacuum gave a colorless viscous liquid that gradually solidified at low temperature (0.60 g, 75\%). ${ }^{1} \mathrm{H}$ NMR (399.952 MHz, $\left.\mathrm{C}_{6} \mathrm{D}_{6}\right): \square=7.61\left(\mathrm{~d},{ }^{3} \mathrm{~J}_{\mathrm{HH}}=8.0 \mathrm{~Hz}, 2 \mathrm{H}\right.$, $\mathrm{Ph}-\mathrm{H} 2,6), 7.13\left(\mathrm{~d},{ }^{3} \mathrm{~J}_{\mathrm{HH}}=8.0 \mathrm{~Hz}, 2 \mathrm{H}, \mathrm{Ph}-\mathrm{H} 3,5\right), 2.63$ (heptet, $\left.{ }^{3} \mathrm{~J}_{\mathrm{HH}}=7.2 \mathrm{~Hz}, 1 \mathrm{H}, \mathrm{CH}\left[\mathrm{CH}_{3}\right]_{2}\right), 1.05$ $\left(\mathrm{d},{ }^{3} \mathrm{~J}_{\mathrm{HH}}=7.2 \mathrm{~Hz}, 6 \mathrm{H}, \mathrm{CH}\left[\mathrm{CH}_{3}\right]_{2}\right) ;{ }^{13} \mathrm{C} \mathrm{NMR}\left(100.564 \mathrm{MHz}, \mathrm{C}_{6} \mathrm{D}_{6}\right): \square=159.4(\mathrm{Ph}-\mathrm{C} 4), 146.5(\mathrm{dm}$, $\left.{ }^{1} \mathrm{~J}_{\mathrm{CF}}=250 \mathrm{~Hz}, \mathrm{Pf}-\mathrm{C} 2,6\right), 142.9\left(\mathrm{dm},{ }^{1} \mathrm{~J}_{\mathrm{CF}}=250 \mathrm{~Hz}, \mathrm{Pf}-\mathrm{C} 4\right), 140.6(\mathrm{Ph}-\mathrm{C} 2,6), 137.7\left(\mathrm{dm},{ }^{1} \mathrm{~J}_{\mathrm{CF}}=250\right.$ $\mathrm{Hz}$, Pf-C3,5), 127.0 (Ph-C3,5), 114.1 (m, Pf-C1), $34.9\left(\boldsymbol{C H}\left[\mathrm{CH}_{3}\right]_{2}\right), 23.3\left(\mathrm{CH}\left[\mathrm{CH}_{3}\right]_{2}\right), \mathrm{Ph}-\mathrm{C} 1$ not observed; ${ }^{19} \mathrm{~F}$ NMR $\left(376.263 \mathrm{MHz}, \mathrm{C}_{6} \mathrm{D}_{6}\right): \square=-130.4\left(\mathrm{dd},{ }^{3} \mathrm{~J}_{\mathrm{FF}}=23.0 \mathrm{~Hz},{ }^{4} \mathrm{~J}_{\mathrm{FF}}=9.2 \mathrm{~Hz}, 4 \mathrm{~F}\right.$, Pf-F2,6), -149.3 (t, $\left.{ }^{3} \mathrm{~J}_{\mathrm{FF}}=23.0 \mathrm{~Hz}, 2 \mathrm{~F}, \mathrm{Pf}-\mathrm{F} 4\right),-161.3\left(\mathrm{dt},{ }^{3} \mathrm{~J}_{\mathrm{FF}}=23.0 \mathrm{~Hz},{ }^{4} \mathrm{~J}_{\mathrm{FF}}=9.2 \mathrm{~Hz}, 4 \mathrm{~F}\right.$, Pf-F3,5); ${ }^{11} \mathrm{~B}$ NMR (160.370 MHz, $\left.\mathrm{C}_{6} \mathrm{D}_{6}\right): \square=62\left(\mathrm{w}_{1 / 2}=1,100 \mathrm{~Hz}\right) ; \mathrm{GC}-\mathrm{MS}(\mathrm{t}=10.1 \mathrm{~min}): \mathrm{m} / \mathrm{z}$ : $464\left[\mathrm{M}^{+}\right], 449\left[\mathrm{M}^{+}-\mathrm{CH}_{3}\right], 326\left[\mathrm{M}^{+}\right.$-cumene-F], 277; elemental analysis: calcd. F 40.93; found $\mathrm{F}$ 40.46 .

\section{Additional References}

(1) Eaborn, C. J. Chem. Soc. 1956, 4858.

(2) For the related phenylbis(pentafluorophenyl)borane see: Deck, P. A.; Beswick, C. L.; Marks, T. J. J. Am. Chem. Soc. 1998, 120, 1772.

(3) For the reactivity of $\left[\mathrm{CuC}_{6} \mathrm{~F}_{5}\right]_{4}$ (toluene $)_{2}$ toward boron halides see: Sundararaman, A.; Jäkle, F. J. Organomet. Chem. 2003, 681, 134. 